\title{
TERAPIA OCUPACIONAL UTILIZANDO EL ABORDAJE DE INTEGRACIÓN SENSORIAL: ESTUDIO DE CASO ÚNICO
}

\author{
OCCUPATIONAL THERAPY USING A SENSORY INTEGRATION FRAME OF \\ REFERENCE: SINGLE CASE STUDY
}

\section{Sara Jorquera , Dulce María Romero²}

\begin{abstract}
Resumen:
Objetivo: Estudiar la efectividad de tratamiento desde Terapia Ocupacional utilizando el abordaje de Integración Sensorial ( T.O./I.S). Método: Se ha utilizado un diseño de caso único para tratar de verificar el impacto del tratamiento T.O./I.S en la funcionalidad y participación del niño seleccionado para el estudio. Para la medición de los objetivos se han utilizado entrevistas con los padres, la metodología Goal Attainment Scaling (GAS) y test estandarizados.

Resultados: Tras cinco meses de intervención y realizando 2 sesiones a la semana de cincuenta minutos se logran mejorías en destrezas como, movimientos de anticipación, equilibrio y visomotoras (medidas con test estandarizados), así como en el desempeño ocupacional a nivel escolar, en alimentación y relaciones sociales.

Conclusión: Los resultados apoyan el uso del abordaje de IS, interrelacionando el procesamiento sensorial, el comportamiento y el desempeño ocupacional, tal y como postuló Ayres. Los resultados se basan en los fundamentos teóricos de la teoría de la integración sensorial: la mejora en la capacidad de procesar e integrar la información sensorial influirá en la conducta adaptada y el desempeño. Aunque estos resultados no pueden generalizarse, proporcionan evidencia preliminar sobre la teoría y la eficacia de este enfoque.
\end{abstract}

\section{Palabras Clave:}

Efectividad, integración sensorial, desempeño, terapia ocupacional, participación

\begin{abstract}
Objective: study the effectiveness of treatment from occupational therapy using sensory integration approach (OT / IS).

Methods: We used a single case design to try to verify the impact of treatment TO / IS on selected functionality and child participation in the study. For the measurement of the objectives they have been used interviews with parents and methodology Goal Attainment Scaling (GAS) and standardized test.

Results: After five months of intervention and doing two sessions a week fifty-minute progress is made not only in skills such as anticipatory movements, balance and visual motor (as measured by standardized test), but also in their occupational performance at school, feeding and social relations.

Conclusion: The results support the use of SI Frame of Reference, interrelating sensory processing, behavior and occupational performance, as postulated Ayres. The findings support the theoretical underpinnings of sensory integration theory: that improvement in the ability to process and integrate sensory input will influence adaptive behavior and occupational performance. Although these findings can not be generalized, they provide preliminary evidence supporting the theory and the effectiveness of this approach.
\end{abstract}

\section{Keywords:}

Effectiveness, sensory integration, performance, occupational therapy, participation

1 Terapeuta Ocupacional. Doctora en Neurociencias. Psicóloga. Universidad de Castilla-La Mancha. Facultad de Terapia Ocupacional, Logopedia y Enfermería. Avda. Real Fábrica de la Seda, s/n. 45600. Talavera de la Reina (Toledo). Spain. Teléfono: +34 925721010 . Ext.:5624

2 Terapeuta Ocupacional. Universidad de Castilla-La Mancha. Facultad de Terapia Ocupacional, Logopedia y Enfermería. Avda. Real Fábrica de la Seda s/n 45600- Talavera de la Reina. España Teléfono: +34 925721010 Ext. 5624 E-mail: Dulce.Romero@uclm.es 


\section{INTRODUCCIÓN}

La Doctora Anna Jean Ayres, introdujo la idea de que el sistema nervioso central procesa e integra sensaciones creando la base sobre la cual se desarrolla el comportamiento funcional. Postuló que la información sensorial es el nutriente del sistema nervioso y que éste responde a esta información con alteraciones en su estructura, función y respuestas en el ambiente. Ella usó el término Integración Sensorial (I.S.) para identificar "el proceso neurológico que organiza las sensaciones del propio cuerpo y del ambiente y hace posible su uso para actuar de manera efectiva en el entorno (Ayres, 1979). La Integración Sensorial es el proceso que permite dar respuestas adaptativas a los desafíos del ambiente como resultado natural del desarrollo típico, facilitando el aprendizaje de habilidades que promueven la participación en actividades con significado y propósito en nuestra vida cotidiana (B. Roley, Schaaf, 2001; Schaaf et al., 2015).

Desde que la teoría fue propuesta por primera vez en 1963, los trabajos de Ayres han continuado desarroIlándose por distintos teóricos, investigadores y clínicos. Todo ello ha producido un incremento de investigaciones en I.S., así como un aumento de la evidencia empírica que incluye nuevos modelos explicativos sobre la influencia de la I.S. en el desarrollo, en la funcionalidad del comportamiento y aprendizaje, así como también en nuevos instrumentos de evaluación del procesamiento sensorial y para la evaluar la intervención, tales como la medida de fidelidad de la intervención en I.S. (Mailloux et al., 2007; Parham et al., 2007; Parham et al., 2011; Schaaf et al., 2014).

La información encontrada en la literatura apoya que cuando estos problemas de I.S. interfieren en el desarrollo de las habilidades del niño para participar en las actividades de su grupo de pares o en sus ocupaciones, se utiliza como abordaje de tratamiento Terapia Ocupacional desde el enfoque de Integración Sensorial (T.O./I.S.) (Watling R, 2011).

El abordaje desde de la teoría de la integración sensorial se ha incorporado desde hace décadas en la praxis cotidiana de los terapeutas ocupacionales (T.O), que trabajan con población infantil y se utiliza dentro del dominio y el ámbito específicos de la profesión (la participación y la salud a partir de la implicación en la ocupación) (Case-Smith, Weaver, \& Fristad, 2015; Roley, Schaaf, 2001; Roley, Bissell, Clark, 2009; Roley, Clark, Bissell, Brayman, 2003; Roley et al., 2015; Schaaf \& Lane, 2015), tal y como se refleja en el Marco de Trabajo para la Práctica de Terapia Ocupacional (en adelante T.O.): Dominio y Proceso $3^{\mathrm{a} E d}$ (AOTA, 2014; Roley, Schaaf, 2001; Roley et al., 2009). Los niños que presentan un desarrollo típico pueden desarrollar las habilidades apropiadas para registrar, modular y discriminar la información sensorial que son esenciales para poder participar e involucrarse en ocupaciones cotidianas (regulación emocional, las habilidades sociales y de juego, así como las destrezas motoras finas y gruesas). Sin embargo, cuando hay problemas para integrar las sensaciones, el niño puede ver limitada su habilidad para prestar atención a las tareas, para desarrollar habilidades sociales, para enfrentarse a las demandas escolares, así como desarrollar autonomía en Actividades de la Vida Diaria (AVD) y participar en actividades sociales y familiares. (Cohn, Kramer, Schub, \& May-Benson, 2014). Es por ello, que el modelo de práctica centrada en la familia es esencial en este tipo de intervenciones (Kingsley \& Mailloux, 2013). La familia será, en la mayoría de las ocasiones, el eslabón entre el entorno clínico y la vida cotidiana del niño. Por ello, a la hora de planificar y valorar los resultados de la intervención necesitamos herramientas que valoren el compromiso con la ocupación y la participación del niño en sus entornos habituales. La presentación de este caso clínico muestra un ejemplo de cómo se pueden medir estas variables utilizando una escala de logro de objetivos, que no solo evalúa resultados de participación del niño en el entorno, sino que además permite guiar el tratamiento desde T.O. sobre el significado y valor que esa ocupación tiene para el niño y su familia. Este trabajo está basado en estudios de caso único realizados en EEUU (Schaaf \& Nightlinger, 2007) y principalmente en la aplicación de esta metodología llamada GAS :Goal Attainment Scaling (Mailloux et al., 2007).

El objetivo de este trabajo es explorar la efectividad del tratamiento desde la Terapia Ocupacional basada en el enfoque de Integración Sensorial en un niño de 6 años que presenta problemas de I.S., con dificultades en su desempeño escolar, social y en las actividades de la vida diaria. 


\section{Método}

\subsection{Participante}

Se trata de un niño " $\mathrm{D}$ " de 6 años y 2 meses en el momento de comenzar la intervención. Vive con sus padres y con su hermano de 2 años. La madre tuvo un embarazo y parto normales. No hay antecedentes médicos de interés. La familia de " $\mathrm{D}$ " se pone en contacto con el Centro de Atención Temprana y Desarrollo Infantil AYTONA por iniciativa propia. Se muestran interesados en que se realice una evaluación para determinar si los problemas de alimentación, de aprendizaje escolar y conductuales que presenta son susceptibles de recibir intervención desde T.O./I.S. Los padres fueron informados de los procedimientos que se iban a realizar durante el proceso de evaluación e intervención y dieron su consentimiento informado por escrito para iniciar el tratamiento, una vez que fue explicado en qué consistiría y la duración del mismo. Las principales preocupaciones de los padres se basan en tres áreas: la alimentación, aprendizaje y rendimiento académico, así como la participación social y conducta.

En relación con la alimentación, " $\mathrm{D}$ " ha tenido problemas con la alimentación desde que se produce la transición de texturas y el cambio de semisólido a sólido. Actualmente su dieta es muy limitada. Según informan los padres, " $\mathrm{D}$ " no tolera determinadas texturas ni la presentación de nuevos alimentos, produciéndose problemas conductuales importantes a la hora de comer. Las preferencias alimenticias del niño son alimentos blandos, de fácil masticación, y crujientes como los empanados. Además, precisa que para masticarlo adecuadamente debe acompañar cada alimento con grandes cantidades de pan. Rechaza las salsas, así como las comidas que impliquen mayor grado de masticación (como la ternera) y mezclas de colores y/o sabores en un mismo plato. Esta limitación ha hecho que " $\mathrm{D}$ " no pudiera acudir al comedor escolar, ya que el niño presentaba cada vez más rechazo a acudir al colegio y comenzaba a tener problemas de peso. El problema se ve agravado cuando la madre tiene que incorporarse de nuevo al trabajo, implicando que el niño tiene debe volver al comedor del colegio.

Por otro lado, " $\mathrm{D}$ " presenta algunas dificultades en el aprendizaje, retraso en la adquisición del lenguaje, que lleva siendo tratado desde hace un año y medio por una logopeda privado, además de recibir media hora a la semana de apoyo en el colegio. La profesora refiere que es un niño lento en los aprendizajes básicos y que parece que presenta problemas atencionales en clase (ha sido derivado para evaluación específica del Equipo de Orientación Psicopedagógica de su zona). Los padres mencionan que no quiere hacer tareas escolares en casa, presentando llantos y quejas en estos momentos, que añadidos a la resistencia a comer, dificultan el manejo conductual del niño en el hogar.

Además, " $\mathrm{D}$ " muestra una disminución en las actividades de participación social y problemas de conducta. Los padres indican que no tiene demasiados amigos, suele ir con otro niño de clase, que también presenta alguna dificultad de desarrollo. La madre indica que el niño parece más infantil que el resto de los niños de su edad. La interacción con los adultos también es limitada, no siendo capaz de entablar conversaciones por iniciativa propia, ni tampoco cuando le preguntan. Su madre refiere que le preocupa que no le cuente lo que hace en el colegio o si tiene algún problema.

\subsection{Instrumentos}

Se han utilizado distintos instrumentos de evaluación:

- Perfil Sensorial (Sensory Profile, SP). Es un cuestionario dirigido a los padres y profesores, que aunque no es una herramienta diagnóstica, sí permite conocer como el niño responde a las distintas demandas sensoriales de su vida diaria , así como las estrategias de auto-regulación que emplea (Dunn, 1999).

- Observación clínica estructurada del procesamiento sensorial. Ayres desarrolló un conjunto de observaciones clínicas estructuradas que están relacionadas al procesamiento sensorial y su efecto sobre el movimiento o comportamiento (Ayres, 1989). Propuso que estas fueran parte de cada evaluación, complementando los resultados de las pruebas estandarizadas. El objetivo es valorar si un rendimiento inadecuado en las tareas cotidianas puede estar relacionado con el procesamiento vestibular, propioceptivo y/o propioceptivo-vestibular (Blanche, 2010; Blanche, Bodison, Chang, \& Reinoso, 2012; Blanche \& Reinoso, 2008).

- Observación no estructurada durante el juego. Se evalúa mediante una situación de juego libre del niño, observando las preferencias sensoriales, prefe- 
rencias y evitaciones lúdicas, las limitaciones funcionales y estrategias utilizadas. Este instrumento está basado en la escala de juego de Knox (Bundy, 2012).

- Movimiento ABC-2. Es un test de desarrollo motor para niños con edades comprendidas entre 3 y 16 años, que mide la destreza manual, las habilidades de recoger y tirar (sacos, pelota) y el equilibrio estático y dinámico (Henderson, Sudgen, \& Barnett, 2012).

- Beery-Buktenica de Integración Viso motora- VMI (5 $5^{\text {a }}$ edición). Es un test de desarrollo de la Integración Visual Motora, que evalúa el grado de integración de las habilidades visuales y motoras de un individuo. Los test de percepción visual y coordinación motora ayudan a comparar los resultados del test con desempeños relativamente puros motores y visuales. Tiene datos normativos para niños desde los 2 años y a adultos hasta los 99 años (Beery, Buktenica, \& Beery, 2010).
- Escala de logros (Goal Attainment Scaling, GAS). Es una metodología desarrollada inicialmente en el campo de la enfermedad mental por Kiresuk y Sherman (Kiresuk \& Sherman, 1968). La metodología GAS es congruente con la filosofía de T.O. centrada en el cliente, ya que identifica los resultados de la intervención que son especialmente relevantes para el individuo, sus familias y su entorno. Este concepto de GAS se utiliza para: (a) predecir el nivel de desempeño esperado en el niño después de un específico periodo de tiempo; (b) identificar de forma graduada los incrementos sobre y por debajo del nivel de desempeño esperado. Es una escala de 5 puntos $(-2 \mathrm{a}+2)$, que permite diferenciar entre el valor peor de lo esperado (-2), al valor esperado (0), o mejor de lo esperado (+2) (ver Tabla 1). Se puede consultar en el Anexo II la aplicación de la Escala GAS en el caso de estudio.

- Entrevista con los padres, que está basada la escala para el establecimiento de objetivos GAS (Mailloux et al., 2007)

Tabla 1

Escala de Niveles de GAS (7).

\begin{tabular}{|c|c|}
\hline Valor & Descripción \\
\hline-2 & $\begin{array}{r}\text { Mucho menos que el resultado esperado: Este nivel refleja que el desempeño esperado se produce el } 7 \% \text { del tiem- } \\
\text { po, va hacia la regresión o mínimos cambios. }\end{array}$ \\
\hline-1 & $\begin{array}{r}\text { Algo menos del resultado esperado: Este nivel refleja que el desempeño ocurre } 21 \% \text { de las veces y es menos de lo } \\
\text { esperado para el tiempo de intervención. }\end{array}$ \\
\hline 0 & Desempeño esperado al final del período : este nivel indica el nivel de desempeño esperado y ocurre el $43 \%$ del tiempo \\
\hline+1 & $\begin{array}{r}\text { Mejor que el esperado: Este nivel refleja que el desempeño ocurre aproximadamente un } 21 \% \text { del tiempo e indica } \\
\text { que hay más progreso del esperado durante el período de intervención }\end{array}$ \\
\hline+2 & $\begin{array}{r}\text { Mucho más que el esperado: Este nivel refleja que el desempeño ocurre aproximadamente un } 7 \% \text { del tiempo y es } \\
\text { inusual porque significa más progreso que el esperado durante el tiempo de medición }\end{array}$ \\
\hline
\end{tabular}

\section{Procedimiento}

Se ha utilizado un diseño de caso único para tratar de verificar el impacto del tratamiento T.O./I.S en la funcionalidad y participación del niño seleccionado para el estudio. En el proceso ha participado un T.O. clínico encargado de llevar a cabo la evaluación inicial y el tratamiento del niño y el T.O. investigador que se encargó del establecimiento de objetivos mediante la escala GAS junto con la familia. El procedimiento Ilevado a cabo tras seleccionar el caso, fue el siguiente:

- Recogida de información a través de los instrumentos mencionados en el apartado anterior (llevado a cabo por el T.O clínico). 
- Análisis de los datos obtenidos (T.O clínico y T.O investigador).

- Planificación de los objetivos de intervención junto con los padres (padres y T.O investigador).

- Establecimiento de un período de intervención de cinco meses (20 semanas), con una frecuencia de dos sesiones semanales de cincuenta minutos (llevado a cabo por el T.O clínico).

- Medición y supervisión de objetivos semanal (padres y T.O investigador).

- Evaluación post-tratamiento del niño (T.O clínico).

- Análisis cualitativo de los datos y elaboración de conclusiones (T.O investigador).

A continuación, se indican los objetivos de tratamiento por orden de prioridad para los padres:

Objetivo 1: Participar en una variedad de actividades motoras orales en base a aumentar su repertorio en alimentación e incrementar su participación en actividades relacionadas (comedor del colegio, comidas familiares, cumpleaños, etc.)

Objetivo 2 : Mejorar el control postural y el equilibrio de manera que pueda mantener la postura de sedestación en las horas de comida/clase y aumente el tiempo de atención en las tareas.

Objetivo 3: Mejorar el desarrollo motor fino, para que pueda participar en las actividades escolares y de manipulación en las actividades de la vida diaria.

Objetivo 4: Mejorar las praxis y el desarrollo motor apropiado para su edad que le permitan su participación en la actividades escolares y de juego.

Objetivo 5: Mejorar las habilidades sociales y de comunicación, aumentando la interacción con los adultos y su grupo de pares.

Basándonos en los objetivos planteados se realizó la siguiente planificación de tratamiento:
- Asistencia a la clínica dos días en semana en sesiones de 50 minutos, para recibir tratamiento de T.O./I.S. (A continuación se explica en líneas generales como se llevó a cabo el tratamiento en sala).

- Recomendaciones o estrategias sensoriales para casa y colegio.

La intervención en las dificultades de discriminación de la información sensorial usando este enfoque de I.S. se dirigió a proveer a " $\mathrm{D}$ " de oportunidades que proporcionaran un desarrollo de la discriminación táctil, vestibular y propioceptiva con el fin de incrementar la conciencia corporal. Este feedback sensorial aportaría una planificación de movimientos más precisa en el espacio y en relación con otros objetos en el ambiente. Se hizo especial hincapié en cuanto a la discriminación táctil, involucrándole en una variedad de experiencias táctiles de cuerpo entero, así como actividades orales, que le proporcionasen información a las áreas corticales de asociación que interpretan estas experiencias sensitivas. Los movimientos memorizados se almacenan para poder ser usados en un futuro en el diseño y planificación de otros movimientos.

La intervención en cuanto al control postural, se dirigió a proveer a " $\mathrm{D}$ " de actividades ricas en información vestibular y propioceptiva junto con información visual, que le ayudaran a mejorar el equilibrio, el control antigravitatorio, la extensión prona, la estabilidad de la musculatura del tronco y la habilidad del uso de ambos lados del cuerpo de forma coordinada. Inicialmente, se enfatizó en las habilidades de feedback para pasar a trabajar después en el feedforward y en la secuenciación de actividades, involucrándole en juegos o situaciones que requerían estas habilidades.

La intervención en la praxis, se utilizaron actividades de alta cantidad de input somatosensorial que ayudaron a mejorar la representación de su esquema corporal, se proporcionó un ambiente novedoso con una variedad de formas, tamaños y texturas que le implicasen en nuevos planes motores y en la superación de desafíos de planificación en el espacio, se dio al niño oportunidad de resolver problemas para que pusiese en marcha planes motores generados por sí mismo y se incrementó la variedad de demandas motoras con particular atención en que finalmente lograra la destreza motora. 
La intervención en alimentación, en ambas sesiones, antes de pasar a trabajar actividades propias de la zona oral, se ha utilizado el $60 \%$ del tiempo en actividades sensoriales y motoras en sala, descritas en los apartados anteriores, y el otro $40 \%$ se dedicó expresamente al trabajo de esta área, siguiendo siempre una estructura de actividades preparatorias de la zona oral, con el fin de aumentar la conciencia de retroalimentación de las estructuras orales y que podía consistir en juegos con masticadores, vibradores, juguetes de soplar, pitar, etc.. Y, todo aquello, que implicase la puesta en marcha de habilidades motoras orales (calibración y graduación movimientos mandíbula, coordinación de la lengua, masticación, etc..), combinado con la introducción de nuevos alimentos. Estas sesiones se programaron a última hora de la tarde, de manera que " $\mathrm{D}$ " trabajaba sobre la situación real de la hora de la cena. La T.O. coordinaba semanalmente con los padres el alimento que iba a ser introducido y que protocolo seguir en el domicilio con el niño.

La intervención en Actividades Motoras Finas. La baja discriminación táctil-propioceptiva y el pobre desarrollo de las habilidades visuales motoras hacen que " $\mathrm{D}$ " presente dificultades en escritura, dibujo, hacer puzles, copiar de la pizarra u organizar los materiales. Por ello, la intervención se enfocó a mejorar la manipulación, la aprehensión de objetos, los movimientos aislados de los dedos, el uso bilateral de ambos manos y el uso de herramientas como tijeras, lápices, cubiertos, etc. En parte de las sesiones, se hizo hincapié en actividades de estiramientos de las manos, apoyo y descarga de peso en las manos, uso de materiales con resistencia (plastilina, tijeras, pinzas), actividades de preescritura y actividades manipulativas de la vida diaria como atarse cordones, cremalleras y botones.

La intervención en Habilidades Sociales. La intervención desde T.O., en este aspecto, se dirigió a fomentar la comunicación y la interacción social-comunicativa del niño a través de situaciones lúdicas, que le impliquen en juegos simbólicos y sociales más cercanos a su edad de desarrollo, donde compartiese tareas y juegos con otros niños que están en la sala de tratamiento de forma cooperativa. Se integró a los padres en parte de las sesiones para que adquiriesen habilidades de manejo de conducta y ampliar el repertorio de juegos que ayudasen a " $D$ " a relacionarse con pares y familia en entornos naturales, con el fin de generalizar las habilidades adquiridas en la sala de tratamiento.

\section{Resultados}

La evaluación inicial desde T.O. nos indica que " $\mathrm{D}$ " presenta problemas de integración sensorial que se han constatado a través de las observaciones clínicas estructuradas, los cuestionarios que fueron completados por los padres y por los resultados obtenidos en los test administrados.

\subsection{Se adjuntan a modo de resumen los hallazgos encontrados:}

Según los resultados del Perfil Sensorial (Sensory Profile), que los padres completaron se han encontrado resultados significativos diferentes a la norma en: procesamiento vestibular, táctil, multisensorial, sensorialoral, modulación de los input sensoriales relacionados con el tono muscular, la resistencia física, (modulación de estímulos propioceptivos y vestibulares) bajo tono muscular, tendencia a la distracción y en respuestas emocionales ligadas al input sensorial.

En cuanto al resultado de las observaciones clínicas estructuradas efectuadas, el niño presentó problemas en la realización de algunas pruebas en referencia a su edad:

- A nivel propioceptivo: Diadococinesis, toque secuencial de los dedos, dedo-nariz, movimientos lentos en arco y test de Schilder.

- A nivel vestibular: Extensión en prono, estabilidad visual y nistagmus postrotario. En conjunto a nivel vestibular y propioceptivo tuvo problemas en llevar a cabo el skipping y saltos, así como en las pruebas posturales con ojos cerrados.

- A nivel táctil: problemas en la detección y localización de estímulos táctiles.

En la observación no estructurada durante el juego: Se observaron dificultades en el manejo del espacio, sobre todo en lo referente a la actividad motora gruesa con pobre control postural y de equilibrio, escasa estabilidad articular, sobreuso de la visión para imitar cosas simples, así como tendencia a caerse con frecuencia y problemas de anticipación para atrapar recoger objetos. En cuanto al manejo del material e imitación, presenta poca exploración, se limita a los materiales 
o equipo que la terapeuta le sugiere, sugiriendo pobre ideación y planeamiento motor. En cuanto a participación, se observa un juego insuficientemente elaborado para su edad, escaso uso del lenguaje y de cooperación con la T.O.

Los resultados de los test estandarizados se muestran en los Gráficos 1 y 2, en los que se compara con la puntuación del niño con la puntuación media de niños de su edad y con la puntuación obtenida tras la intervención T.O./I.S.

\subsection{Resultados tras período de intervención.}

Se han comparado los resultados del Beery-VMI obtenidos en Diciembre del 2012, al comienzo de la intervención, y en Abril de 2013, tras los cinco meses de tratamiento (Gráfico 1). Se obtienen mejores puntuaciones (en referencia a la media de los niños de su edad cronológica, representado en azul), en el test efectuado en el mes de Abril. Los resultados muestran mejoría significativa, en las funciones de integración visomotoras, de percepción visual y de coordinación motora.

Grafico 1

Resultados Beery-VMI

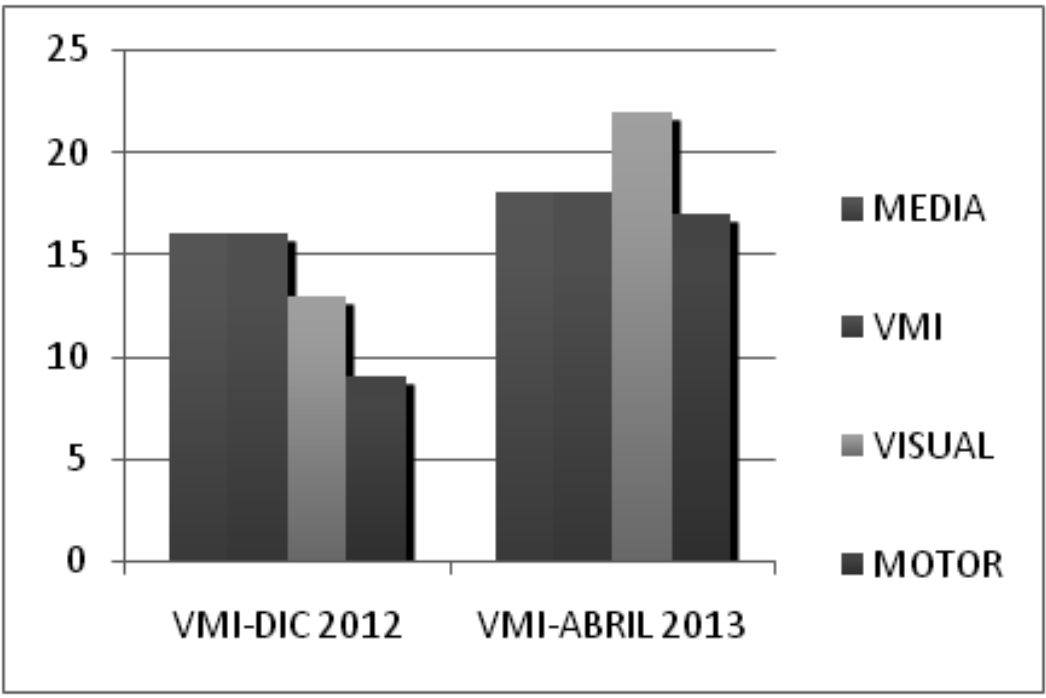

También se muestra una comparativa de las pruebas motoras (Gráfico 2) que se realizan en el test Movimiento $\mathrm{ABC}-2$, comparándose las puntuaciones con respecto a las que obtienen la media de los niños de su edad, mostrándose mejorías en las pruebas de atrapar y soltar (anticipación) y equilibrio, manteniéndose por debajo de la media las puntuaciones en destreza manual.
En cuanto a los datos del GAS completado por los padres, se observa una mejoría en los cinco objetivos propuestos, se presentan los resultados (Gráfico 3) a lo largo de las 20 semanas de tratamiento. 
Gráfico 3

Resultados de GAS ( Goal Attainment Scaling)

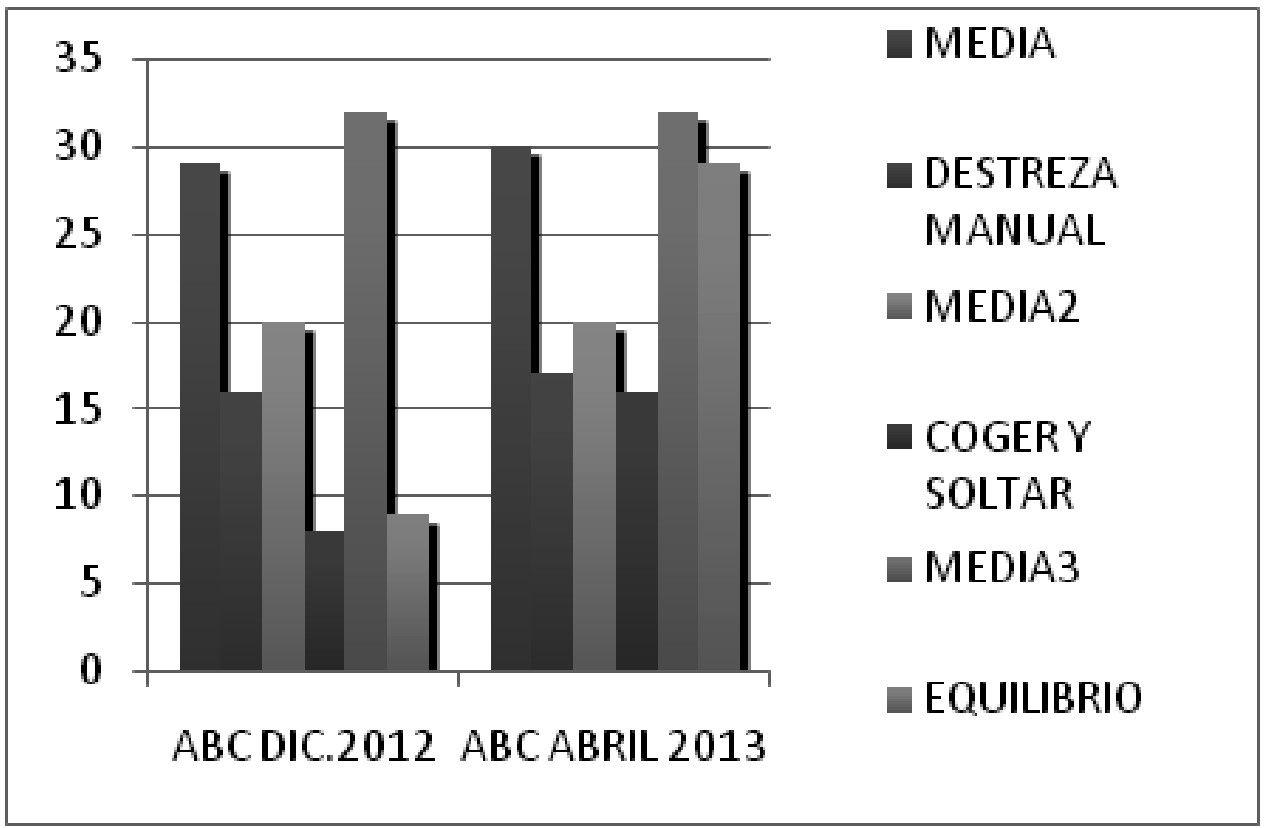

\section{DisCUSIÓN}

Los resultados obtenidos apoyan los principios del uso de este marco de I.S., interrelacionando el procesamiento sensorial, la conducta y el desempeño ocupacional, tal y como postuló Ayres.

En el caso de estudio se observa progreso en el área de alimentación, el niño amplía el repertorio de alimentos, aparecen cambios positivos de conducta durante las cenas familiares y mayor predisposición a acudir al comedor escolar. Se denotan aparentes avances en los tiempos de atención y mantenimiento de actividades en mesa, así como en los juegos con pares y desarrollo de la interacción social. Tanto en tratamiento como en casa se observan mejorías en la exploración y participación de actividades visuales motoras y motoras finas, aunque estos resultados no se reflejan en la medición de destreza motora fina del MABC-2., que sigue siendo un objetivo a trabajar, y sería importante trasladar la medición de este objetivo al ambiente escolar, de cara a obtener información acerca de la generalización de logros. El tratamiento se basó en el grado de importancia que los padres dieron a los objetivos planteados en la escala GAS, siendo el objetivo de más peso la alimentación, por ello otros objetivos siguen en proceso.

Los resultados obtenidos en GAS en cuanto a la participación del niño deben ser interpretados con cautela, ya que representan los objetivos relevantes para la familia de " $\mathrm{D}$ ", en particular, por lo que no se pueden generalizar a otros casos. Serían necesarias otras medidas estandarizadas de evaluación del desempeño ocupacional para ser utilizadas como medidas pretest y postest.

Como ventajas del uso de GAS, destacar que nos proporciona una guía para planificar, identificar y monitorizar los objetivos de intervención, así como los resultados en T.O. GAS permite a los padres comprender los problemas reales del niño así como sus progresos de una manera gradual. Recientes estudios han encontrado que aunque los padres observen cambios en la parte sensorial o motora que se reflejan en los test estandarizados, ellos dan más valor a los aspectos funcionales no medidos en los test tradicionales (Cohn, Miller, \& Tickle, 2000). La escala GAS permite recoger el progreso individualizado que es significativo para la familia, siendo una herramienta válida para el uso durante 
y después del tratamiento T.O.-I-S, tanto en la clínica como en la investigación (Mailloux et al., 2007).

En cuanto a las limitaciones del estudio, la principal es que es un estudio $n=1$, por lo que no se pueden extrapolar resultados al resto de la población. Además, no se han aplicado pruebas estadísticas para estimar si los cambios son estadísticamente significativos. Por otro lado, tan solo se han medido las respuestas de los padres en cuanto a impacto del tratamiento en el entorno familiar, sería interesante poder recoger las opiniones del colegio y del comedor escolar. Otra limitación es la referida a la validez y confiabilidad de alguno de los instrumentos de recolección de datos utilizados (Sensory Profile), ya que, no está adaptado en la población española. Para una mayor fuerza estadística sería recomendable comenzar a crear o en su defecto adaptar transculturalmente otras medidas de evaluación del desempeño ocupacional, como Miller Function and Participation Scale (Miller, 2006).

Como futuros estudios sería recomendable un estudio similar con mayor muestra o en este aspecto también sería interesante conocer los efectos a largo plazo de estas adquisiciones mediante un estudio de cohortes, así como poder realizar estudios con mayor nivel de evidencia científica, tales como ensayos clínicos, con grupo de comparación y asignación aleatoria.

Agradecimientos: Nuestro más sincero agradecimiento al niño y a su familia por su colaboración y a la Terapeuta Ocupacional clínica Elena Y.G.

\section{REFERENCIAS BIBLIOGRÁFICAS}

aOTA, American Occupational Therapy Association. (2014). Occupational Therapy practice framework :Domain and process (3rd ed.) (Vol. 68 (Suppl.1), pp. S1-S4B): American Journal of Occupational Therapy.

Ayres, A. J. (1979). Sensory Integration and the child. Los Angeles, CA: Westren Psychological Services.

Ayres, A. J. (1989). Sensory integration and Praxis Test. Los Angeles: Western Psychological Services.

Beery, K., Buktenica, N. , Beery, N. (2010). VMI: Beery-Buktenica Developmental Test of Visual-Motor Integration (6th ed. ed.). Minneapolis: Pearson.

Blanche, E. I. (2010). Observations based on sensory integration theory. Torrance, CA.: Pediatric Therapy Network.
Blanche, E. I., Bodison, S., Chang, M. C., Reinoso, G. (2012). Development of the comprehensive observations of proprioception (COP): validity, reliability, and factor analysis. Am / Occup Ther, 66(6), 691-698. doi:10.5014/ajot.2012.003608

Blanche, E., Reinoso, G. (2008). The use of clinical observations to evaluate proprioceptive and vestibular functions. OT Practice, 13(17), CE1-CE6.

Bundy, A. (2012). Can I play Too? In Shelly J Lane \& Anita C Bundy (Eds.), Kids can be Kids. Philadelphia: FA Davis.

Case-Smith, J., Weaver, L. L., Fristad, M. A. (2015). A systematic review of sensory processing interventions for children with autism spectrum disorders. Autism, 19(2), 133-148.

Cohn, E., Miller, L., Tickle-Degnen, L. (2000). Parental hopes for therapy outcomes: Children with sensory modulation disorders. American Journal of occupational Therapy(54), 36-43.

Cohn, E. S., Kramer, J., Schub, J. A., May-Benson, T. (2014). Parents' explanatory models and hopes for outcomes of occupational therapy using a sensory integration approach. Am J Occup Ther, 68(4), 454-462.

Dunn, W. (1999). The Sensory Profile: User's manual. San Antonio: The Psychological Corporation.

Henderson, S, Sudgen, D, Barnett, A. (2012). MABC-2.Bateria de Evaluación del Movimiento para niños. Barcelona: Pearson.

Kingsley, K., Mailloux, Z. (2013). Evidence for the effectiveness of different service models in early intervention services. American Journal of Occupational Therapy, 64(7), 431-436.

Kiresuk, T. J., Sherman, R. E. (1968). Goal attainment scaling: A general method for evaluating comprehensive community mental health programs. Community Ment Health J, 4(6), 443-453.

Mailloux, Z., May-Benson, T. A., Summers, C. A., Miller, L. J., BrettGreen, B., Burke, J. P., Schoen, S. A. (2007). Goal attainment scaling as a measure of meaningful outcomes for children with sensory integration disorders. Am J Occup Ther, 61(2), 254-259.

Miller, LJ. (2006). Miller Function and participation scale.Mm. USA: Pearson.

Parham, L. D., Cohn, E. S., Spitzer, S., Koomar, J. A., Miller, L. J., Burke, J. P., Summers, C. A. (2007). Fidelity in sensory integration intervention research. Am J Occup Ther, 61(2), 216-227.

Parham, L. D., Roley, S. S., May-Benson, T. A., Koomar, J., Brett-Green, B., Burke, J. P., Schaaf, R. C. (2011). Development of a fidelity measure for research on the effectiveness of the Ayres Sensory Integration intervention. Am J Occup Ther, 65(2), 133-142.

Roley, Blanche, Schaaf. (2001). Sensory Integration with diverse population (Pro-ed Ed.). Austin, Texas.

Roley, S. S., Bissell, J., Clark, G. F., (2009). Providing occupational therapy using sensory integration theory and methods in school-based practice. Am J Occup Ther, 63(6), 823-842. 
Roley, S. S., Clark, G. F., Bissell, J., Brayman, S. J., (2003). Applying sensory integration framework in educationally related Occupational Therapy practice (2003 statement). Am J Occup Ther, 57(6), 652-659.

Roley, S. S., Mailloux, Z., Parham, L. D., Schaaf, R. C., Lane, C. J., Cermak, S. (2015). Sensory integration and praxis patterns in children with autism. Am J Occup Ther, 69(1), doi:10.5014/ajot.2015.012476

Schaaf, R. C., Burke, J. P., Cohn, E., May-Benson, T. A., Schoen, S. A., Roley, S., Mailloux, Z. (2014). State of measurement in occupational therapy using sensory integration. Am J Occup Ther, 68(5), e149153. doi:10.5014/ajot.2014.012526

Schaaf, R., Lane, A. (2015). Toward a Best-Practice Protocol for Assessment of Sensory Features in ASD. J Autism Dev Disord, 45(5), 1380-1395.

Schaaf, R., Nightlinger, K. (2007). Occupational therapy using a sensory integrative approach: a case study of effectiveness. Am J Occup Ther, 61(2), 239-246.

Schaaf, R., Schoen, S., May-Benson, T., Parham, D., Lane, S., Roley, S., Mailloux, Z. (2015). State of the Science: A Roadmap for Research in Sensory Integration. Am J Occup Ther, 69(6), doi:10.5014/ ajot.2015.019539.

Watling R., Patten Koening K., Davies P., Schaaf R. (2011). Occupational Therapy Practice Guidelines for children and adolescents with chaIlenges in Sensory Processing and Sensory Integration. Bethesda: AOTA Press. 